\title{
Características Antropométricas de los Jugadores Españoles de Voley Playa. Comparación por Categorías
}

\author{
Anthropometric Characteristics of Spanish Beach Volleyball Players. Comparison by Categories
}

Miriam Esther Quiroga Escudero; Samuel Sarmiento Montesdeoca*; Antonio Palomino Martín*; David Rodríguez Ruiz* \& Juan Manuel García Manso*

QUIROGa, M. E.; SARMIENTO, S.; PALOMINO, A.; RODRÍGUEZ-RUIZ, D. \& GARCÍA-MANSO, J. M. Características antropométricas de los jugadores españoles de voley playa. Comparación por categorías. Int. J. Morphol., 32(1):22-28, 2014.

RESUMEN: El objetivo de este estudio fue determinar las características antropométricas del jugador español (hombres y mujeres) de voley playa y comparar el perfil antropométrico de estos deportistas en las diferentes categorías en las que se organiza la competición: Sub-19, Sub-21 y Absoluto. Fueron evaluados 150 jugadores (79 hombres y 71 mujeres) participantes en el Campeonato de España de voley playa celebrado en 2011. La estatura presentó valores que responden, en los hombres, a patrones evolutivos propios de edad y morfotipo



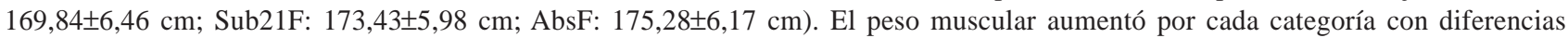
estadísticamente significativas entre AbsM vs. Sub19M ( $p=0,002)$, AbsM vs. Sub21M ( $p=0,001)$ y Sub21F vs. Sub19F ( $p=0,02)$. Los valores de peso corporal y porcentaje graso mostraron diferencias menos relevantes con diferencias estadísticamente significativas entre el grupo Sub21F ( $\mathrm{p}=0,003$ ) y el Sub19F ( $\mathrm{p}=0,009$ ). Los jugadores españoles de voley playa Absolutos, tanto hombres como mujeres, presentan un peso corporal y una estatura inferior a la de los jugadores de alto nivel internacional. El peso muscular y el peso graso muestran en ambos sexos, en valores absolutos, valores crecientes con cada categoría de edad, pero no se confirma esta tendencia cuando el parámetro se expresa en porcentajes respecto al peso corporal. El jugador de voley playa tiende a incrementar el valor de la mesomorfia con el cambio de categoría, tanto en hombres $(3,33 \pm 1,09 ; 3,77 \pm 1,28 ; 4,28 \pm 1,07)$ como en mujeres $(2,75 \pm 1,02 ; 2,86 \pm 1,21 ; 3,36 \pm 0,92)$.

PALABRAS CLAVE: Voley playa; Composición corporal; Somatotipo.

\section{INTRODUCCIÓN}

El voley playa fue jugado por primera vez en las playas de Hawái y de California (Estados Unidos) en 1920, y no es hasta 1996 que se convierte en disciplina olímpica en los Juegos Olímpicos celebrados en Atlanta. Su creciente proyección internacional y su evolución técnico-táctica han despertado el interés de técnicos e investigadores para mejorar las estrategias de entrenamiento e incrementar el rendimiento. Sin embargo, en la actualidad siguen existiendo pocos estudios científicos que centren su atención en esta modalidad deportiva (Ricarte Batista et al., 2008). Esto conlleva que sean escasos los trabajos que han examinado la composición corporal de los jugadores de voley playa (Medeiros et al., 2010; Martínez-Sanz et al., 2010a, 2010b, 2010c).

El análisis del perfil antropométrico ayuda a caracterizar las reservas deportivas de esta modalidad y a mejorar y especializar los procesos de entrenamiento en función de las características de juego y el rol que tiene asignado cada juga- dor. Existen estudios realizados en voleibol comparando diferentes niveles o categorías (Gualdi-Russo \& Zaccagni, 2001; Gabbett \& Georgieff, 2007; Fonseca et al., 2010), pero no conocemos ningún trabajo que haya estudiado el perfil corporal del jugador de voley playa en sus diferentes categorías de edades. En esta línea, Medeiros et al., proponen evaluar los datos de la composición corporal en jugadores de voley playa de diferentes países y niveles de competición para, de esta forma, poder elaborar una base de datos con la que poder caracterizar a estos deportistas y permitir así la identificación de marcadores específicos. En nuestro caso, conocer los datos antropométricos de los jugadores de voley playa españoles nos permitirá caracterizar esta muestra para su comparación con otros deportistas del mismo rendimiento. Dentro de la composición corporal, el tejido muscular y graso son componentes que pudieran intervenir en el rendimiento de los jugadores. El somatotipo es otro factor que nos ayuda a distinguir las formas de las muestras.

\footnotetext{
* Departamento de Educación Física, Universidad de Las Palmas de Gran Canaria (ULPGC), Islas Canarias, España.
} 
El objetivo de este estudio ha sido conocer las características antropométricas del jugador español (masculino y femenino) de voley playa y comparar el perfil antropométrico de estos deportistas en las diferentes categorías en las se organiza la competición: Sub-19, Sub-21 y Absoluto.

\section{MATERIAL Y MÉTODO}

La muestra estuvo formada por 150 jugadores (hombres y mujeres) participantes en los Campeonatos de España de voley playa (Sub-19, Sub-21 y Absoluto) celebrados en Las Palmas de Gran Canaria (2011). Del total de jugadores evaluados, 29 pertenecían a la categoría Absoluta masculina (AbsM), 11 eran jugadoras Absolutas femeninas (AbsF), 26 Sub-21 masculinos (Sub21M), 28 Sub-21 femeninos (Sub21F), 24 jugadores Sub-19 masculinos (Sub19M) y 32 Sub-19 femeninos (Sub19F) (Tabla I). Todos los sujetos fueron informados de la naturaleza del estudio y de sus intenciones de uso y realizaron un escrito con su consentimiento, de acuerdo con las líneas éticas de la declaración de Helsinki, para la investigación con seres humanos (Adoptado por la 18 Asamblea Médico Mundial de Helsinki en 1964 y modificado por la 59 Asamblea General celebrada en Seúl en 2008).
Se utilizó una báscula marca Detecto (Lafayette Instruments Company, Lafayette, Indiana, USA), cuyo intervalo de medición se sitúa entre 0 y $150 \mathrm{~kg}$ y nivel de precisión de 200 g. Se realizaron calibraciones periódicas, así como ajustes del cero, previo a cada medición. Para la medición de la altura corporal de cada sujeto, se empleó un estadiómetro Holtain (Holtain Ltd., Dyfed, UK) con rango de precisión de $1 \mathrm{~mm}$. Los pliegues de grasa subcutánea del tríceps, subescapular, suprailiaco, abdominal, anterior del muslo, y pierna fueron evaluados con un lipómetro Holtain Skinfold Caliper (Holtain Ltd., Dyfed, UK) con amplitud de 0 a $48 \mathrm{~mm}$, graduación de $0,2 \mathrm{~mm}$ y presión constante de $10 \mathrm{~g} / \mathrm{mm} 2$ (Carter \& Heath, 1990). Los perímetros del brazo contraído, brazo relajado, cintura, muslo y de la pierna se midieron con una cinta métrica metálica, flexible pero inextensible, (Holtain Ltd., Dyfed, UK) de escala 0,1 cm. Finalmente, los diámetros biepicondilar del húmero, bicondilar del fémur, y el biestiloideo se obtuvieron con un paquímetro convencional de cremallera (Holtain Ltd, Crymych, Reino Unido), con plano triangular de broca para la cabeza, cuyo rango de medida abarca desde 63 a $213 \mathrm{~cm}$, con un error de medida de $\pm 0,1 \mathrm{~mm}$. Con los datos obtenidos se determinaron los valores de peso graso, muscular y óseo, los porcentajes de tejido adiposo, muscular y óseo, así como el cálculo del somatotipo.

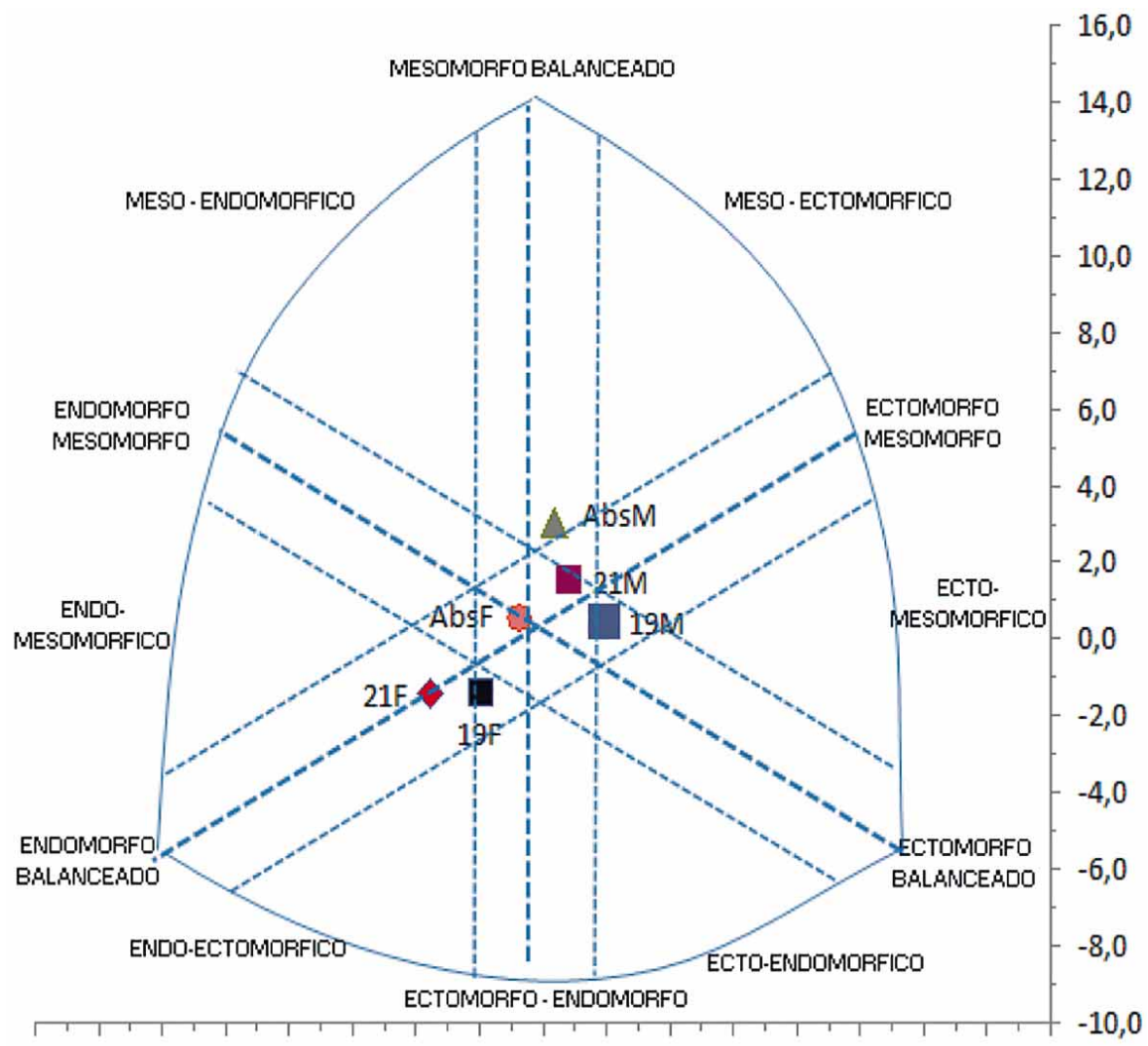

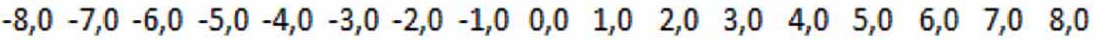

Fig. 1. Somatocarta por categorías y sexo. 
La descripción de puntos anatómicos, masa corporal, perímetros, diámetros y pliegues cutáneos, así como la metodología llevada a cabo en la toma de datos, corresponde a la desarrollada por Lohman et al. (1988). Para el cálculo del Índice de Masa Corporal (IMC) se empleó la fórmula de Quetelet utilizada por Garrow \& Webster (1985). Para la valoración del somatotipo utilizamos la metodología descrita por Heath-Carter (Carter, 1975), mientras que para el estudio de la composición corporal nos basamos en la propuesta presentada por De Rose \& Guimaraes (1980) a partir de la ecuación de Matiegka (1921), determinando el porcentaje de grasa por la ecuación de Carter (1982). La masa ósea se halló por la ecuación de Rocha (1975). Atendiendo las recomendaciones y la metodología de diversos autores (Durnin \& Rahaman, 1967; Martorell et al., 1988; Housh et al., 1990), las mediciones se realizaron en el lado dominante del deportista.

Para el análisis estadístico de los datos obtenidos de la muestra de estudio se usó el paquete SPSS para Windows versión 19.0 (SPSS Inc., Chicago, IL, USA). La caracterización de la muestra y en la descripción de las variables del estudio se llevó a cabo una estadística descriptiva básica por categoría y género. Se aplicó una prueba de normalidad (Shapiro-Wilk) y para la comparación de medias se utilizó una t-Student y la U de Mann-Whitney para series paramétricas o no paramétricos (nivel de significación de $\mathrm{p} \leq 0,05$ ).

\section{RESULTADOS}

En la Tabla I se muestran la media y desviación estándar de la edad y de los datos antropométricos básicos, la talla y el peso corporal (PC), así como la media y desviación estándar del índice de masa corporal (IMC) de la muestra de estudio por categorías tanto a nivel masculino como femenino.

En la Tabla II se detallan los valores promedios y desviación estándar de los perímetros registrados (brazo flexionado, cintura, muslo y pierna), así como los diáme-

Tabla I. Caracterización de la muestra por categorías y sexo.

\begin{tabular}{lcccccc}
\hline & \multicolumn{3}{c}{ Masculino $(\mathbf{n}=\mathbf{7 9})$} & \multicolumn{3}{c}{ Femenino $(\mathbf{n = 7 1})$} \\
\cline { 2 - 7 } & $\begin{array}{c}\text { Sub19 } \\
(\mathbf{n = 2 4})\end{array}$ & $\begin{array}{c}\text { Sub 21 } \\
(\mathbf{n = 2 6})\end{array}$ & $\begin{array}{c}\text { Absoluto } \\
(\mathbf{n = 2 9})\end{array}$ & $\begin{array}{c}\text { Sub 19 } \\
(\mathbf{n = 3 2})\end{array}$ & $\begin{array}{c}\text { Sub 21 } \\
(\mathbf{n = 2 8})\end{array}$ & $\begin{array}{c}\text { Absoluto } \\
(\mathbf{n = 1 1})\end{array}$ \\
\hline \multirow{2}{*}{ Edad (años) } & 16,96 & 19,00 & 29,76 & 16,34 & 18,86 & 29,18 \\
& $( \pm 0,86)$ & $( \pm 0,94)$ & $( \pm 5,65)$ & $( \pm 1,04)$ & $( \pm 1,15)$ & $( \pm 4,69)$ \\
Talla (cm) & 184,50 & 182,89 & 186,93 & 169.84 & 173,43 & 175,28 \\
& $( \pm 6,95)$ & $( \pm 7,28)$ & $( \pm 6,58)$ & $( \pm 6,46)$ & $( \pm 5,98)$ & $( \pm 6,17)$ \\
PC (kg) & 75,43 & 75,65 & 83,24 a & 62,14 & 67,49 & 67,46 \\
& $( \pm 9,24)$ & $( \pm 10,96)$ & $( \pm 6,46)$ & $( \pm 7,78)$ & $( \pm 8,05)$ & $( \pm 6,64)$ \\
IMC $\left(\mathrm{kg} / \mathrm{m}^{2}\right)$ & 22.13 & .65 & 23.83 & 21.50 & 22.44 & 21.95 \\
& $( \pm 2,10)$ & $( \pm 3,32)$ & $( \pm 1,45)$ & $( \pm 1,97)$ & $( \pm 2,32)$ & $( \pm 1,71)$ \\
\hline
\end{tabular}

(a) $\mathrm{p} \leq 0,01$ (AbsM vs. Sub19M).

Tabla II. Descriptivo de los valores antropométricos por categorías y sexo.

\begin{tabular}{lcccccc}
\hline & \multicolumn{3}{c}{ Masculino $(\mathbf{n = 7 9})$} & \multicolumn{3}{c}{ Femenino $(\mathbf{n = 7 1})$} \\
\cline { 2 - 6 } & $\begin{array}{c}\text { Sub 19 } \\
(\mathbf{n = 2 4})\end{array}$ & $\begin{array}{c}\text { Sub 21 } \\
(\mathbf{n = 2 6})\end{array}$ & $\begin{array}{c}\text { Absoluto } \\
(\mathbf{n = 2 9})\end{array}$ & $\begin{array}{c}\text { Sub 19 } \\
(\mathbf{n = 3 2})\end{array}$ & $\begin{array}{c}\text { Sub 21 } \\
(\mathbf{n = 2 8})\end{array}$ & $\begin{array}{c}\text { Absoluto } \\
(\mathbf{n = 1 1})\end{array}$ \\
\hline \multirow{2}{*}{ Brazo Flexionado (cm) } & 30,33 & 31,00 & 32,62 & 26,53 & 26,29 & 27,36 \\
& $( \pm 2,51)$ & $( \pm 2,51)$ & $( \pm 2,34)$ & $( \pm 1,87)$ & $( \pm 4,81)$ & $( \pm 0,92)$ \\
Cintura (cm) & 76,58 & 78,12 & 85,66 a & 70,97 & 76,39 b & 82,91 a \\
& $( \pm 5,59)$ & $( \pm 7,69)$ & $( \pm 3,18)$ & $( \pm 7,24)$ & $( \pm 8,76)$ & $( \pm 6,52)$ \\
Muslo (cm) & 53,92 & 53,81 & 56,97 b & 51,69 & 53,96 & 55,18 \\
& $( \pm 4,17)$ & $( \pm 4,39)$ & $( \pm 2,46)$ & $( \pm 3,33)$ & $( \pm 3,38)$ & $( \pm 2,56)$ \\
Pierna (cm) & 37,63 & 37,31 & 37,62 & 34,88 & 35,61 & 36,55 \\
& $( \pm 2,67)$ & $( \pm 2,31)$ & $( \pm 1,86)$ & $( \pm 2,11)$ & $( \pm 2.28)$ & $( \pm 2,16)$ \\
Biepicondilar Húmero $(\mathrm{cm})$ & 6,96 & 7,00 & 7,28 & 6,06 & 6,18 & $6,55 \mathrm{a}$ \\
& $( \pm 0,20)$ & $( \pm 0,40)$ & $( \pm 0,46)$ & $( \pm 0,44)$ & $( \pm 0,48)$ & $( \pm 0,52)$ \\
Bicondilar Fémur $(\mathrm{cm})$ & 9,96 & 10,00 & 10,31 & 9,03 & 9,32 & 9,64 \\
& $( \pm 0,75)$ & $( \pm 0,49)$ & $( \pm 0,47)$ & $( \pm 0,60)$ & $( \pm 0,72)$ & $( \pm 0,67)$ \\
Biestiloideo (cm) & 5.79 & 5.88 & 5.97 & 5.09 & 5.00 & 5.18 \\
& $( \pm 0,42)$ & $( \pm 0,77)$ & $( \pm 0,19)$ & $( \pm 0,39)$ & $( \pm 0,27)$ & $( \pm 0,41)$ \\
\hline
\end{tabular}

(a) $\mathrm{p} \leq 0,01$ (AbsM vs. Sub19M, AbsF vs. Sub19F). (b) $\mathrm{p} \leq 0,05$ (AbsM vs Sub19M, Sub21F vs. Sub19F). 
tros (biepicondilar del húmero, bicondilar del fémur y biestiloideo) de las categorías masculinas y femeninas respectivamente. Podemos observar que los perímetros no mantienen el incremento de cada categoría como pudiera parecer lógico con el incremento de la edad, comportamiento que sí se produce en los diámetros con la excepción mencionada del grupo Sub21 femenino.
En la Tabla III se reflejan los datos de composición corporal (peso muscular, óseo y graso expresado en $\mathrm{kg} \mathrm{y}$ porcentaje frente al PC) por categorías y sexo. La muestra masculina, en el componente de masa muscular y grasa, describe incrementos por categorías que luego no se mantienen con sus porcentajes. La muestra femenina no mantiene, por su grupo Sub-21, una tónica lineal.

Tabla III. Descriptivo de los valores de composición corporal por categorías y sexo.

\begin{tabular}{|c|c|c|c|c|c|c|}
\hline & \multicolumn{3}{|c|}{ Masculino $(n=79)$} & \multicolumn{3}{|c|}{ Femenino $(n=71)$} \\
\hline & $\begin{array}{c}\text { Sub 19 } \\
(n=24)\end{array}$ & $\begin{array}{c}\text { Sub 21 } \\
(n=26)\end{array}$ & $\begin{array}{c}\text { Absoluto } \\
(\mathbf{n}=29)\end{array}$ & $\begin{array}{r}\text { Sub 19 } \\
(n=32)\end{array}$ & $\begin{array}{c}\text { Sub 21 } \\
(n=28)\end{array}$ & $\begin{array}{c}\text { Absoluto } \\
(n=11)\end{array}$ \\
\hline Peso Muscular (kg) & $\begin{array}{c}37,64 \\
( \pm 4,35)\end{array}$ & $\begin{array}{c}37,73 \\
( \pm 4,73)\end{array}$ & $\begin{array}{c}41,22 \mathrm{a} \\
( \pm 3,53)\end{array}$ & $\begin{array}{c}28,64 \\
( \pm 3,08)\end{array}$ & $\begin{array}{c}30,02 \\
( \pm 3.35)\end{array}$ & $\begin{array}{c}30,69 \\
( \pm 3,58)\end{array}$ \\
\hline$\%$ Muscular & $\begin{array}{c}49,97 \\
( \pm 1,38)\end{array}$ & $\begin{array}{c}50,02 \\
( \pm 2,08)\end{array}$ & $\begin{array}{c}49,53 \\
( \pm 1,92)\end{array}$ & $\begin{array}{c}46,22 \\
( \pm 2,18)\end{array}$ & $\begin{array}{l}44,60 \mathrm{~b} \\
( \pm 2.72)\end{array}$ & $\begin{array}{c}45,47 \\
( \pm 2,67)\end{array}$ \\
\hline Peso Óseo (kg) & $\begin{array}{c}12,59 \\
( \pm 1,43)\end{array}$ & $\begin{array}{c}12,53 \\
( \pm 1,62)\end{array}$ & $\begin{array}{c}13,71 \mathrm{a} \\
( \pm 1,13)\end{array}$ & $\begin{array}{c}9,59 \\
( \pm 1,16)\end{array}$ & $\begin{array}{c}10,16 \\
( \pm 0,98)\end{array}$ & $\begin{array}{l}11,06 \mathrm{a}^{\mathrm{b}} \\
( \pm 1,05)\end{array}$ \\
\hline \% Óseo & $\begin{array}{c}16,77 \\
( \pm 1,57)\end{array}$ & $\begin{array}{c}16,72 \\
( \pm 2,07)\end{array}$ & $\begin{array}{c}16,49 \\
( \pm 1,10)\end{array}$ & $\begin{array}{c}15,48 \\
( \pm 1,24)\end{array}$ & $\begin{array}{c}15,13 \\
( \pm 1,25)\end{array}$ & $\begin{array}{c}16,42 \mathrm{a} \\
( \pm 0,77)\end{array}$ \\
\hline Peso Graso (kg) & $\begin{array}{c}7,02 \\
( \pm 2,01)\end{array}$ & $\begin{array}{c}7,16 \\
( \pm 3,59)\end{array}$ & $\begin{array}{c}8,26 \mathrm{a}^{\mathrm{b}} \\
( \pm 1,83)\end{array}$ & $\begin{array}{c}10,92 \\
( \pm 2,74)\end{array}$ & $\begin{array}{l}13,20 \text { a } \\
( \pm 3,31)\end{array}$ & $\begin{array}{c}18,64 \\
( \pm 2,37)\end{array}$ \\
\hline$\%$ Graso & $\begin{array}{c}9,16 \\
( \pm 1,62)\end{array}$ & $\begin{array}{c}9,16 \\
( \pm 3,01)\end{array}$ & $\begin{array}{c}9,88 \mathrm{~b} \\
( \pm 1,86)\end{array}$ & $\begin{array}{c}17,39 \\
( \pm 2,46)\end{array}$ & $\begin{array}{l}19,36 \text { a } \\
( \pm 3,11)\end{array}$ & $\begin{array}{c}17,21 \\
( \pm 2,63)\end{array}$ \\
\hline$\sum 6$ Pliegues (mm) & $\begin{array}{c}62,57 \\
( \pm 15,41)\end{array}$ & $\begin{array}{c}63,70 \\
( \pm 29,62)\end{array}$ & $\begin{array}{c}69,44 \mathrm{~b} \\
( \pm 17,74)\end{array}$ & $\begin{array}{c}89,23 \\
( \pm 15,90)\end{array}$ & $\begin{array}{r}101,96 \text { a } \\
( \pm 20,07)\end{array}$ & $\begin{array}{c}88,06 \text { b } \\
( \pm 17,02)\end{array}$ \\
\hline Endomorfia & $\begin{array}{c}2,63 \\
( \pm 0,65)\end{array}$ & $\begin{array}{c}2,81 \\
( \pm 1,17)\end{array}$ & $\begin{array}{c}2,66 \\
( \pm 0,81)\end{array}$ & $\begin{array}{c}3,94 \\
( \pm 0,80)\end{array}$ & $\begin{array}{c}4,46 \\
( \pm 1,11)\end{array}$ & $\begin{array}{c}3,27 \\
( \pm 0,91)\end{array}$ \\
\hline Mesomorfia & $\begin{array}{c}3,33 \\
( \pm 1,09)\end{array}$ & $\begin{array}{c}3,77 \\
( \pm 1,28)\end{array}$ & $\begin{array}{c}4,28 \\
( \pm 1,07)\end{array}$ & $\begin{array}{c}2,75 \\
( \pm 1,02)\end{array}$ & $\begin{array}{c}2,86 \\
( \pm 1,21)\end{array}$ & $\begin{array}{c}3,36^{c} \\
( \pm 0,92)\end{array}$ \\
\hline Ectomorf ia & $\begin{array}{c}3.58 \\
( \pm 1,10)\end{array}$ & $\begin{array}{c}3.23 \\
( \pm 1,58)\end{array}$ & $\begin{array}{c}2.86 \\
( \pm 0,92)\end{array}$ & $\begin{array}{c}2.97 \\
( \pm 1,09)\end{array}$ & $\begin{array}{c}2.68 \\
( \pm 1,19)\end{array}$ & $\begin{array}{c}2.91 \\
( \pm 1,04)\end{array}$ \\
\hline
\end{tabular}

(a) $\mathrm{p} \leq 0,01$ (AbsF vs Sub19F; Sub19F vs Sub21F; AbsF vs Sub21F; AbsM vs Sub19M; AbsM vs Sub21M).

(b) $\mathrm{p} \leq 0,05$ (Sub21F vs. Sub19F; AbsF vs Sub21F; AbsM vs Sub21M; AbsF vs. Sub19F).

c) $\mathrm{p} \leq 0,05$ (AbsF vs. Sub19F).

La representación de las diferentes categorías de la muestra queda reflejada en la somatocarta (Fig. 1).

\section{DISCUSIÓN}

La distribución de la muestra por niveles de competición (Sub-19, Sub-21 y Absoluta) nos permite observar que la estatura se incrementa con la edad y el morfotipo de los jugadores masculinos (Sub19M: 184,50 $\pm 6,95 \mathrm{~cm}$; Sub21M: 182,89 $\pm 7,28 \mathrm{~cm}$; AbsM, 186,93 $\pm 6,58 \mathrm{~cm}$ ), pero en las mujeres no se mantiene este comportamiento (Sub19F:


$175,28 \pm 6,17 \mathrm{~cm}$ ) debido al grupo Sub21F.
Comparando estos resultados con los trabajos que realizaron Medeiros et al., con jugadores brasileños de edad similar (Absolutos), se observa como los jugadores españoles son significativamente más bajos $(7,3 \mathrm{~cm})$ que los brasileños, siendo este parámetro un factor determinante del rendimiento en esta modalidad deportiva. Si los comparamos con los datos de jugadores/as que participaron en el World Tour y los JJ.OO. entre las temporadas 2000 y 2006 aportados por Palao et al. (2008), vemos como se repite esta circunstancia con valores más elevados para los jugadores/as de mayor nivel (Diferencia Masculina: 6,07 cm; Diferencia Femenina: $3,72 \mathrm{~cm}$ ). Ricarte Batista et al. dividen en dos grupos los jugadores de elite masculinos brasileños $\left(1^{\circ}-7^{\circ} \mathrm{y}\right.$ $8^{\circ}-10^{\circ}$ puesto), comprobándose que los sujetos de nuestra muestra presentan una estatura inferior frente a los dos gru- 
pos (7,97 y 4,97 $\mathrm{cm}$ respectivamente). Tili \& Giatsis (2011), que estudiaron a 91 jugadores masculinos de 16 países de la FIVB de voley playa, muestran resultado que, nuevamente, son superiores $(5,77 \mathrm{~cm})$ a los de nuestro estudio. En cambio, Giatsis et al. (2011), al estudiar 56 finalistas de la FIVB de voley playa, constatan que las diferencias respecto a nuestra muestra de la categoría femenina presentan diferencias menos relevantes $(3,52 \mathrm{~cm})$ que las encontradas en los jugadores masculinos.

En relación al peso corporal, los hombres presentan mínimas diferencias entre los grupos Sub-19M y Sub-21M, pero sí diferencias importantes con la categoría AbsM (7,8 $\mathrm{kg}$ con Sub-19M y 7,5 kg con Sub-21M) con una diferencia significativa $(\mathrm{p}=0,014)$ respecto a los grupos AbsM y Sub19M. Sin embargo, en las mujeres si existe una diferencia mayor entre la categoría Sub-19F y Sub-21F, aunque no estadísticamente significativa.

No hay diferencias entre Sub-21 y Absoluta. Según los resultados de Palao et al., nuestros jugadores tienen un peso inferior en varones $(5,76 \mathrm{~kg})$ y mujeres $(0,54 \mathrm{~kg})$. Cuando comparamos estos datos con los aportados por Ricarte Batista et al., también se cumple este comportamiento (Puesto $1^{\circ}-7^{\circ}: 8,06 \mathrm{~kg}$; Puesto $8^{\circ}-10^{\circ}: 4,36 \mathrm{~kg}$ ).

La mayor parte de los estudios epidemiológicos utilizan el IMC para evaluar el grado de obesidad de la muestra objeto de estudio. Se acepta que a mayores valores de IMC, mayores niveles de obesidad, en el caso de poblaciones de deportistas, este criterio no se ajusta a los valores de referencia que suelen emplearse con sujetos sedentarios. El IMC de nuestra muestra describe una progresión ascendente en relación a las categorías, sin diferencias significativas. La excepción se establece, nuevamente, en el grupo Sub21F $\left(22,44 \pm 2,32 \mathrm{~kg} / \mathrm{m}^{2}\right)$ con valores superiores de IMC que las jugadoras absolutas (diferencia promedio de $0,49 \mathrm{~kg} / \mathrm{m}^{2}$ ). En relación a los trabajos de Palao et al., en categoría absoluta nuestra muestra masculina presentan unos valores menores de $0,27 \mathrm{~kg} / \mathrm{m}^{2}$ y superiores en el grupo femenino (de $\left.0,85 \mathrm{~kg} / \mathrm{m}^{2}\right)$.

El peso muscular aumenta con la edad (Tabla III) destacando la existencia de diferencias estadísticamente significativas entre AbsM y los Sub19M $(7,81 \mathrm{~kg} ; \mathrm{p}=0,002)$ y entre AbsM y Sub21M (7,59 kg; p=0,001). En la muestra femenina las diferencias se establecen en el porcentaje muscular Sub21F con Sub19F $(1,62 \%$; p=0,02). Nuevamente, estos valores no reflejan la continuidad que esperamos en estas edades, debido a las características de la muestra Sub21F evaluada.

Los valores de peso corporal y porcentaje graso fe- menino muestran diferencias significativas al comparar el grupo Sub21F con Sub19F (5,35 kg; p=0,003 y 1,97\%; $\mathrm{p}=0,009$, respectivamente). Esto lo ratifica el valor de sumatorio de pliegues $(12,73 \mathrm{~mm} ; \mathrm{p}=0,008)$ lo que nos indica que dicho grupo (Sub21F) presenta valores altos de este componente y bajos o relativos en el muscular, justo lo menos conveniente para deportistas de este nivel competitivo. A su vez, el sumatorio de pliegues de las absolutas femeninas mantienen diferencias con las $\operatorname{Sub} 21 \mathrm{~F}(13,9$ $\mathrm{mm} ; \mathrm{p}=0,04)$. Esto nos indica que en relación a las jugadoras Sub21F, las AbsF presentaron una mejor composición corporal, mayor tejido muscular y menor tejido graso. En relación al componente graso masculino mostraron diferencias significativas entre los absolutos y los Sub19M $(1,24$ kg; $\mathrm{p}=0,02)$ y con los Sub21M $(1,1 \mathrm{~kg}$.; $\mathrm{p}=0,004)$ pero sólo en la masa porque en porcentaje las diferencias se establecen con el grupo Sub21M (0,72\%; p=0,033), este valor se refrenda con el sumatorio de pliegues que muestra diferencias con el mismo grupo $(5,74 \mathrm{~mm} ; \mathrm{p}=0,04)$. Comparando el porcentaje de grasa de nuestros jugadores masculinos con los de Medeiros et al., de jugadores brasileños, observamos que nuestra muestra tiene un porcentaje mayor de un $1,1 \%$, demostrando que los jugadores evaluados en este estudio están dentro de los márgenes de los jugadores internacionales.

Una forma más específica de caracterizar la muestra suele ser la determinación del somatotipo y su ubicación dentro de la somatocarta. Los valores del somatotipo masculino presentaron un comportamiento similar, destacando en el grupo absoluto el incremento de la mesomorfia, pero en ningún caso con diferencias significativas. Pero que, como mostramos anteriormente, si existe diferencia significativa a la hora de comparar el peso muscular de la composición corporal entre el grupo absoluto con Sub19M y con Sub21M. En cambio, en las jugadoras de nuestra muestra se confirma los datos obtenidos en la composición corporal, en concreto en masa y porcentaje graso, obtenemos como el grupo Sub21F tiene valores superiores en la endomorfia, porque muestran diferencias estadísticamente significativas en el peso y porcentaje graso con los otros dos grupos. Las diferencias significativas las hemos encontrado en el grupo absoluto en relación al $\operatorname{Sub19F}(0,61 ; p=0,04)$ en la mesomorfia, debido a que la masa y porcentaje óseo de absolutas son significativamente mayores que las que muestran las Sub19F. La muestra masculina tiende de la ectomesomorfia a una mesomorfia balanceada. En cambio la muestra femenina su comportamiento fue irregular, por el grupo Sub21F, pero entre el Sub19F y absoluto la tendencia fue a la mesomorfia balanceada. De forma general, el jugador de voley playa con el incremento de la edad, y por tanto de la categoría, tiene una tendencia al aumento de los valores de mesomorfia. 
En los jugadores de voley playa masculinos, la estatura, el peso corporal y el IMC responden a los patrones normales de aumento con la edad y el desarrollo del morfológico propio de un practicante de esta modalidad deportiva. Los jugadores de voley playa Absolutos, masculinos y femeninos, presentan pesos y alturas inferiores a los jugadores de categoría internacional, comparado con los trabajos de otros autores. El peso muscular y el graso también muestran valores crecientes tanto en categoría masculina como femenina. La muestra masculina Absoluta establece diferencias significativas con las otras dos categorías tanto en peso muscular como graso. En cambio cuando estudiamos dichos componentes en valores porcentuales este incremento no se confirma. Al com- parar con los trabajos de otros autores, la muestra del estudio, presenta valores más bajos en peso corporal, porcentajes mayores en grasa y menores en tejido muscular.

\section{AGRADECIMIENTOS}

Agradecemos la colaboración prestada por la Federación Canaria de Voleibol para la realización de este estudio durante el transcurso de los Campeonatos de España de Voley Playa celebrados en Las Palmas de Gran Canaria en julio de 2011.

QUIROGA, M. E.; SARMIENTO, S.; PALOMINO, A.; RODRÍGUEZ-RUIZ, D. \& GARCÍA-MANSO, J. M. Anthropometric characteristics of Spanish beach volleyball players. Comparison by category. Int. J. Morphol., 32(1):22-28, 2014.

SUMMARY: The objective of this study was to determine the anthropometric characteristics of Spanish beach volleyball players (men and women) and compare their anthropometric profile across competition categories: Under 19, Under 21 and Senior. A total of 150 players (79 men and 71 women) participating in the 2011 Spanish Beach Volleyball Championship were assessed. Height values in male players corresponded to development patterns appropriate to age and morphotype (U19M: 184,50 $\pm 6,95 \mathrm{~cm} ; \mathrm{U} 21 \mathrm{M}$ : $182 \pm 7,28 \mathrm{~cm}$;

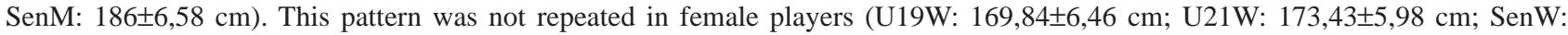
$175,28 \pm 6,17 \mathrm{~cm}$ ). Muscle weight increased by category, with statistically significant differences between SenM vs. U19M ( $\mathrm{p}=0,002)$, SenM vs. U21M ( $\mathrm{p}=0,001)$ and U21W vs. U19W ( $\mathrm{p}=0,02)$. Body weight and fat percentage values showed less pronounced differences, with statistically significant differences between groups U21W ( $\mathrm{p}=0,003)$ and U19W ( $\mathrm{p}=0,009)$. Spanish Senior beach volleyball players, both men and women, have lower values for body weight and height than international players. In absolute values, muscle weight and fat weight in both sexes showed increasing values by age category, but this pattern was not confirmed when the parameter was expressed in percentages in relation to body weight. Beach volleyball players tend to increase their mesomorphy value with changes in category, both for men $(3,33 \pm 1,09 ; 3,77 \pm 1,28 ; 4,28 \pm 1,07)$ and women $(2,75 \pm 1,02 ; 2,86 \pm 1,21 ; 3,36 \pm 0,92)$.

KEY WORDS: Beach volleyball; Body composition; Somatotype.

\section{REFERENCIAS BIBLIOGRÁFICAS}

Carter, J. E. L. The Heath-Carter somatotype method. San Diego, San Diego State University, 1975.

Carter, J. E. L. Body Composition of Montreal Olympic Athletes. In: Carter, J. E. L. (Ed.). Physical Structure of Olympic Athletes Part I. Basel, Karger, 1982. pp.107-16.

Carter, J. E. L. \& Heath, B. H. Somatotyping: development and applications. New York, Cambridge University Press, 1990.

De Rose, E. H. \& Guimaraes, A. C. A model for optimization of somatotype in young athletes. In: Ostyn, M.; Buenen, G. \& Simons, J. (Eds.). Kinanthropometry II. Baltimore, University Park Press, 1980. pp.77-80.

Durnin, J. V. \& Rahaman, M. M. The assessment of the amount of fat in the human body from measurements of skinfold thickness. Br. J. Nutr., 21(3):681-9, 1967.

Fonseca, C. L. T.; Fernandes, R. P. \& Fernandes-Filho, J. Analysis of Anthropometrical Profile of Brazilian Junior Volleyball
Team. Int. J. Morphol., 28(4):1035-41, 2010.

Gabbett, T. \& Georgieff, B. Physiological and Anthropometric Characteristics of Australian Junior Nacional, State, and Novice Volleyball Players. J. Strength Cond. Res., 21(3):902-8, 2007.

Garrow, J. S. \& Webster, J. Quetelet's index (W/H2) as a measure of fatness. Int. J. Obes., 9(2):147-53, 1985.

Giatsis, G.; Tili, M. \& Zetou, E. The height of the women's winners FIVB Beach Volleyball in relation to specialization and court dimensions. J. Hum. Sport Exerc., 6(3):497-503, 2011.

Gualdi-Russo, E. \& Zaccagni, L. Somatotype, role and performance in elite volleyball players. J. Sports Med. Phys. Fitness, 41(2):256-62, 2001.

Housh, T. J.; Johnson, G. O.; Housh, D. J.; Kenney, K. B.; Hughes, R. A.; Thorland, W. G. \& Cisar, C. J. The effects of age and body weight on anthropometric estimations of minimal wrestling weight in high school wrestlers. Res. Q. Exerc. Sport, 61(4):375-82, 1990. 
QUIROGA, M. E.; SARMIENTO, S.; PALOMINO, A.; RODRÍGUEZ-RUIZ, D. \& GARCÍA-MANSO, J. M. Características antropométricas de los jugadores españoles de voley playa. Comparación por categorías. Int. J. Morphol., 32(1):22-28, 2014.

Lohman, T. G.; Roche, A. \& Martorell, R. Anthropometric standardization reference manual. Champaign, Human Kinetics, 1988.

Matiegka, J. The testing of physical efficiency. Am. J. Phys. Anthrop., 4(3):223-30, 1921.

Martínez-Sanz, J. M.; Pérez-Turpin, J. A.; Sánchez-García, L. F.; Urdampilleta, A.; Gómez-Zorita, S. \& Cejuela-Anta, R. Antropometría y composición corporal en jugadores de VoleyPlaya del Campeonato Nacional Universitario de Alicante 2010. Arch. Med. Dep., XXVII (139):412-3, 2010 a.

Martínez-Sanz, J. M.; Pérez-Turpin, J. A.; Cejuela-Anta, R.; Sánchez-García, L. F. \& Urdampilleta, A. Composición corporal de jugadores de élite de Voley-Playa. Arch. Med. Dep., XXVII (139):414-5, 2010b.

Martínez-Sanz, J. M.; Cejuela, R.; Pérez-Turpin, J. A.; Chinchilla, J. J.; Cortell-Tormo, J. M. \& Sánchez-García, L. F. Somatotipo en jugadores de élite de Voley-Playa. Arch. Med. Dep., XXVII (139):415, 2010c.

Martorell, R.; Mendoza, F.; Mueller, W. H. \& Pawson, Y. G. Which side to measurement. In: Lohman, T. G., Roche, A. F. \& Martorell, R. (Eds.). Anthropometric standardization reference manual. Champaign, Human Kinetics, 1988. pp.87-92.

Medeiros, A.; Mesquita, I.; Oliveira, J.; Loureiro, A. C. C.; Afonso, J.; Monteiro, L. Z. \& Castro, J. M. Body composition of Brazilian beach volleyball players. Br. J. Sports Med., 44(14): i17, 2010 .

Palao, J. M.; Gutiérrez, D. \& Frideres, J. E. Height, weight, body mass index, and age in beach volleyball players in relation to level and position. J. Sports Med. Phys Fitness, 48(4):466-71, 2008.

Ricarte Batista, G.; Freire De Araújo, R. \& Oliveira Guerra, R. Comparison between vertical jumps of high performance athletes on the Brazilian men's beach volleyball. J. Sports Med. Phys. Fitness, 48(2):172-6, 2008.

Rocha, M. S. L. Peso ósseo do brasileiro de ambos os sexos de 17 a 25 años. Arq. Anat. Antrop., 1:445-51, 1975.

Tili, M. \& Giatsis, G. The height of the men's winners FIVB Beach Volleyball in relation to specialization and court dimensions. J. Hum. Sport Exerc., 6(3):504-10, 2011.
Dirección para Correspondencia:

Miriam Esther Quiroga Escudero

Departamento de Educación Física

Universidad de Las Palmas de Gran Canaria (ULPGC)

Islas Canarias

ESPAÑA

Email: mquiroga@def.ulpgc.es

Recibido : 11-06-2013

Aceptado: 19-11-2013 